\title{
ReCount: A multi-experiment resource of analysis-ready RNA-seq gene count datasets
}

\author{
Alyssa C Frazee, Ben Langmead ${ }^{*}$ and Jeffrey T Leek $^{*}$
}

\begin{abstract}
1 Background: RNA sequencing is a flexible and powerful new approach for measuring gene, exon, or isoform expression. To maximize the utility of RNA sequencing data, new statistical methods are needed for clustering, differential expression, and other analyses. A major barrier to the development of new statistical methods is the lack of RNA sequencing datasets that can be easily obtained and analyzed in common statistical software packages such as R. To speed up the development process, we have created a resource of analysis-ready RNA-sequencing datasets.

2 Description: ReCount is an online resource of RNA-seq gene count tables and auxilliary data. Tables were built from raw RNA sequencing data from 18 different published studies comprising 475 samples and over 8 billion reads. Using the Myrna package, reads were aligned, overlapped with gene models and tabulated into gene-by-sample count tables that are ready for statistical analysis. Count tables and phenotype data were combined into Bioconductor ExpressionSet objects for ease of analysis. ReCount also contains the Myrna manifest files and R source code used to process the samples, allowing statistical and computational scientists to consider alternative parameter values.

3 Conclusions: By combining datasets from many studies and providing data that has already been processed from. fasta format into ready-to-use. RData and. txt files, ReCount facilitates analysis and methods development for RNA-seq count data. We anticipate that ReCount will also be useful for investigators who wish to consider crossstudy comparisons and alternative normalization strategies for RNA-seq.
\end{abstract}

\section{Background}

RNA-seq, or short-read sequencing of mRNA, has emerged as a powerful and flexible tool for studying gene expression [1]. As with other new technologies, the analysis of RNA-seq data requires the development of new statistical methods. Data from many RNA-seq experiments are publicly available, but processing raw data into a form suitable for statistical analysis remains challenging [2]. This difficulty together with the high cost of using secondgeneration sequencing technology means that most computational scientists have only a limited number of samples to work with [3]. However, replication is critical to understanding biological variation in RNA-sequencing [4].

The Gene Expression Omnibus [5] is a useful repository that contains both processed and raw microarray data, but there is no comparable resource for processed RNA-seq data. We have compiled a resource, called ReCount, consisting of aligned, preprocessed RNA-seq

\footnotetext{
* Correspondence: blangmea@jhsph.edu; jleek@jhsph.edu

Department of Biostatistics, The Johns Hopkins University Bloomberg School of Public Health, 615 North Wolfe Street, Baltimore, MD 21205, USA
}

data from 475 samples in 18 different experiments. Our database makes it easier for statistical and bioinformatics researchers to analyze RNA-seq count data using standard tools such as R, Bioconductor [6], and MATLAB. The aligned and preprocessed data in ReCount can be directly analyzed, used to develop and compare new methods for analysis, or examined to identify cross-study effects. The ReCount database also contains the Myrna manifest files and R source code used to process the samples, allowing statistical and computational scientists to consider alternative parameter values.

\section{Construction and Content \\ Content}

We collected data from the 18 experiments described in Table 1 [7-24]. For each experiment, ReCount contains a. txt-format count table encoding, for each sample, the number of reads overlapping each gene included in the Ensembl [25] annotation of the given organism's genome. ReCount also includes manually curated phenotype information (e.g. sex, strain, time point) for each sample,

C Biomed Central

(c) 2011 Frazee et al; licensee BioMed Central Ltd. This is an Open Access article distributed under the terms of the Creative Commons Attribution License (http://creativecommons.org/licenses/by/2.0), which permits unrestricted use, distribution, and reproduction in any medium, provided the original work is properly cited. 
Table 1 Datasets available for download (truncated to $35 \mathrm{bp}$ )

\begin{tabular}{ccrr}
\hline Study & Organism & Number of bio reps & Number of reads \\
\hline BodyMap & human & 19 & $2,197,622,796$ \\
Cheung & human & 41 & $834,584,950$ \\
Core & human & 2 & $8,670,342$ \\
Gilad & human & $41,356,738$ \\
MAQC & human & 6 & $71,970,164$ \\
Montgomery & human & 14 & $* 886,468,054$ \\
Pickrell & human & 60 & $* 886,468,054$ \\
Sultan & human & 69 & $6,573,643$ \\
Wang & human & 4 & $223,929,919$ \\
Katz & mouse & 22 & $14,368,471$ \\
Mortazavi & mouse & 4 & $61,732,881$ \\
Trapnell & mouse & 3 & $111,376,152$ \\
Yang & mouse & 4 & $27,883,862$ \\
Bottomly & mouse & 1 & $343,445,340$ \\
Nagalakshmi & yeast & 21 & $7,688,602$ \\
Hammer & rat & 4 & $158,178,477$ \\
modENCODE - worm & worm & 8 & $1,451,119,823$ \\
modENCODE - fly & fly & 46 & $2,278,788,557$ \\
\hline
\end{tabular}

The "Number of bio reps" column contains the number of individual samples contained in the dataset, while the "Number of reads" column displays the number of uniquely aligned reads that were used to create the count table. A version of this table and an analogous table for the downloadables created by removing Myrna's truncate option are available on the website.

available as a. txt file. Count and phenotype tables were compiled into ExpressionSet objects, which are downloadable from ReCount and can be easily loaded and analyzed using standard Bioconductor tools in R.

\section{Construction}

To construct count tables, we used the Amazon Elastic MapReduce version of Myrna 1.1.2 [26]. As input, Myrna requires a manifest file listing URL locations for all sequencing read files for each sample. Myrna manifest files are available as part of ReCount; most URLs in these files refer to reads stored in the NCBI Sequence Read Archive (SRA) [27].

For studies consisting of paired-end sequencing data, only the first mate of each pair was considered. Many studies also included technical replicates, which were processed using Myrna's pool-tech-reps option. This option pools the reads from technical replicates prior to alignment and analysis. Other options passed to Myrna were bowtie-args = "-v 2-m 1", genefootprint = intersect, and from-middle. The gene-footprint = intersect parameter causes a "union intersection" gene model to be used. The bowtie-args parameters specify that no more than two mismatches are allowed for a read alignment to be valid and that reads with multiple alignments are discarded. The from-middle argument designates that the number of bases considered when overlapping a read's alignment with a gene footprint should be measured from the middle of the read (rather than the 3 ' or 5' end). Finally, we provide count tables and ExpressionSets created using Myrna's truncate $=35$ option, which truncates reads longer than $35 \mathrm{bp}$ to $35 \mathrm{bp}$. For using data from multiple studies at once, the truncation makes studies more comparable to each other; it also decreases the likelihood that a read will span a splice junction and therefore be discarded. However, for researchers who wish to utilize the full read length, we also provide count tables and ExpressionSets created without the truncate option.

Count tables presented in ReCount have not yet been normalized. During analysis, gene counts in each sample are commonly normalized by dividing by the 75 th percentile of the distribution of non-zero gene counts in the sample, as suggested previously [11], but the data presented in ReCount allows researchers to develop, evaluate, and compare alternative normalization schemes.

\section{Utility and Discussion}

\section{User Interface}

The ReCount website features an interactive version of Table 1. ExpressionSets, count tables, and phenotype tables are downloadable from the table. Manifest files used with Myrna, specific Myrna commands used, and R code used to create ExpressionSets are also available for download at the ReCount website. These scripts allow researchers to compare the effects of alternative normalization or alternative Myrna parameterization to the canonical versions of the datasets contained in the ReCount database. The site also contains further details about the contents of the downloadables as well as a set of $\mathrm{R}$ 
commands that may be useful when working with ExpressionSets.

\section{Utility: Example Applications}

ReCount facilitates studies that are not possible using only a small number of samples from a single study. Here we present two toy examples that illustrate the potential utility of the ReCount database. Both examples used datasets created with reads truncated to $35 \mathrm{bp}$.

\section{Application 1: Comparison of normalization methods}

Count tables presented in ReCount have not yet been normalized, which facilitates comparisons between normalization and preprocessing approaches. As an example, we compared 75th percentile normalization [11] with quantile normalization [28] using data collected on two different strains of mice (the bottomly dataset available in ReCount [20]). We analyzed 36,536 total genes, first removing genes with zero counts or that showed no variation across samples (23,697 genes). For both types of normalization, each gene was tested for differential expression between the two strains using an F-test. There were 696 genes that were differentially expressed at a false discovery rate of 5\% (a Benjamini-Hochberg correction for multiple testing [29] was used) in both analyses, while 177 were only differentially expressed using quantile normalization and 35 were only differentially expressed using 75 th percentile normalization. The set of differentially expressed genes for the quantile normalization scheme is a bit larger than the set for the 75th percentile normalization, but the overlap is still quite large. This simple analysis demonstrates a method for comparing normalization schemes; it also illustrates that results of a differential expression analysis differ very little based on which of these two well-established normalization schemes was used.

\section{Application 2: Analysis using data from multiple studies}

Availability of comparable data from many studies facilitates analyses that previously may have been quite cumbersome. As an example, we consider the Cheung [8] and Montgomery [12] data. These two studies assayed 29 of the same individuals. The Cheung group sequenced immortalized B cells, and Montgomery et. al. used lymphoblastoid cell lines, so the types of cells used in sequencing were very similar. Therefore, examining these 29 samples and comparing gene expression between the two studies could provide insight into some of the technical variability present in RNA-seq. As a very basic analysis of differential expression, we compared subjects' measured gene expression in the Cheung study vs. the Montgomery study using a parametric paired t-test on each gene in the table. Genes for which the difference in gene expression was significantly different from zero were considered differentially expressed between studies. (A Benjamini-Hochberg correction for multiple testing was performed; a false-discovery-rate cutoff of 0.05 was used to determine significance). Of the 52,580 genes tested, 3,633 (6.9\%) were found to be differentially expressed between the studies. We also note that 39,752 genes $(75.6 \%)$ could not be tested for differential expression because all counts were zero in both samples. So, of the 12,828 genes that had nonzero gene counts for at least one sample in one of the studies, 3,633 (28.3\%) were differentially expressed. This pattern can be seen in the histogram of the adjusted p-values (Figure 1). This analysis shows evidence of batch-like effects in RNA-seq; differential expression would ideally be quite rare since the same people and similar cell types were analyzed in each study.

As another example of an analysis using multiple datasets found in ReCount, we performed a simple differential expression analysis on the Montgomery data [12] and the Pickrell data [13], which is a proxy for an analysis of differential expression between ethnicities: the Montgomery group sequenced Utah residents with northern- or western-European ancestry (the HapMap CEU population), and the Pickrell group sequenced Yoruba people in Ibadan, Nigeria (the HapMap YRI population). Previous research has addressed this question (e.g. [30,31]), but ReCount facilitates investigation of alternative approaches to the problem. As a starting point, we performed an analysis similar to the previous one: for each of 52,580 genes, we performed a parametric two-sample t-test on the 75thpercentile normalized counts from the Montgomery and

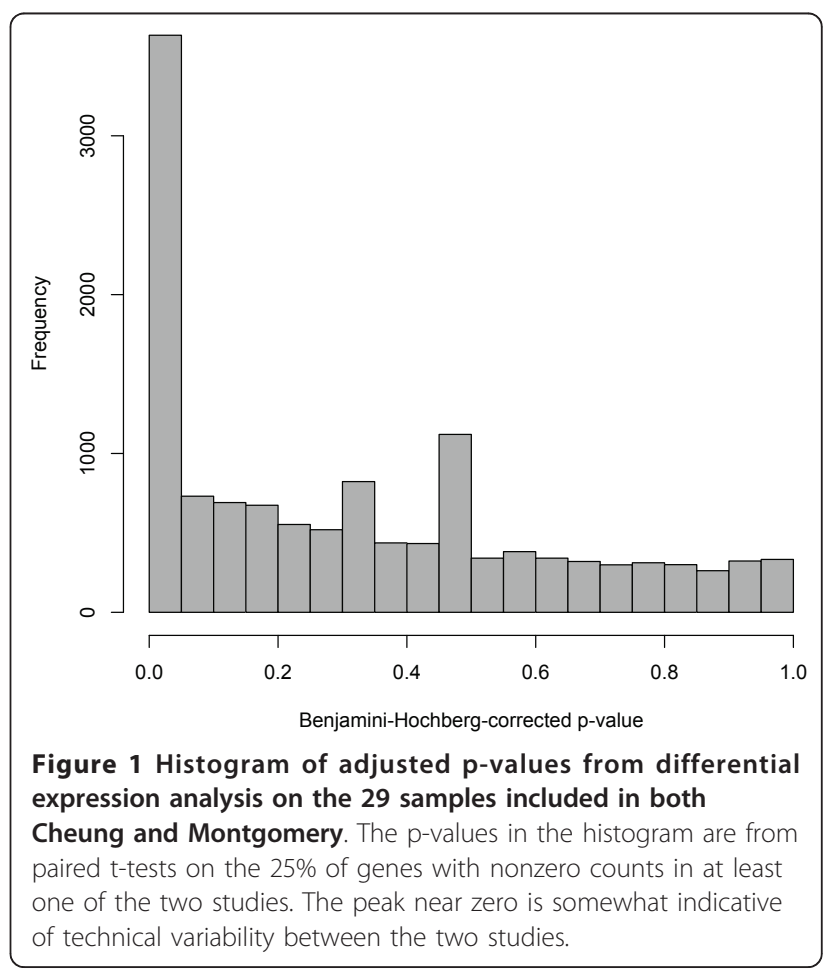


Pickrell data, using a Benjamini-Hochberg correction for multiple testing and considering a gene differentially expressed between CEU and YRI populations if its Benjamini-Hochberg-corrected p-value was less than 0.05. Of the 52,580 genes tested, 4,669 (8.9\%) were found to be differentially expressed. These 4,669 genes comprise $36.0 \%$ of the genes tested for differential expression, i.e., genes with nonzero counts in at least one of the populations (Figure 2). We notice that these percentages are slightly higher than the percentages reported in the previous analysis, which is unsurprising, since both technical and biological variability are present here, whereas the variability in the previous analysis was mostly technical. Follow-up analysis could be performed for these 4,669 genes of interest, e.g., this set could be compared with sets of differentially expressed genes found in previous studies, or expression patterns in individual genes could be visualized. We present these basic analyses as starting points for researchers wishing to simultaneously utilize multiple datasets from ReCount.

\section{Discussion}

ReCount's preproccessed, freely-available data compatible with common statistical software will encourage statisticians interested in methods development to tackle problems arising in RNA-seq data analysis. By providing a large amount of RNA-seq data in a central, accessible location, ReCount facilitates analyses like those above and several others. For example, another interesting

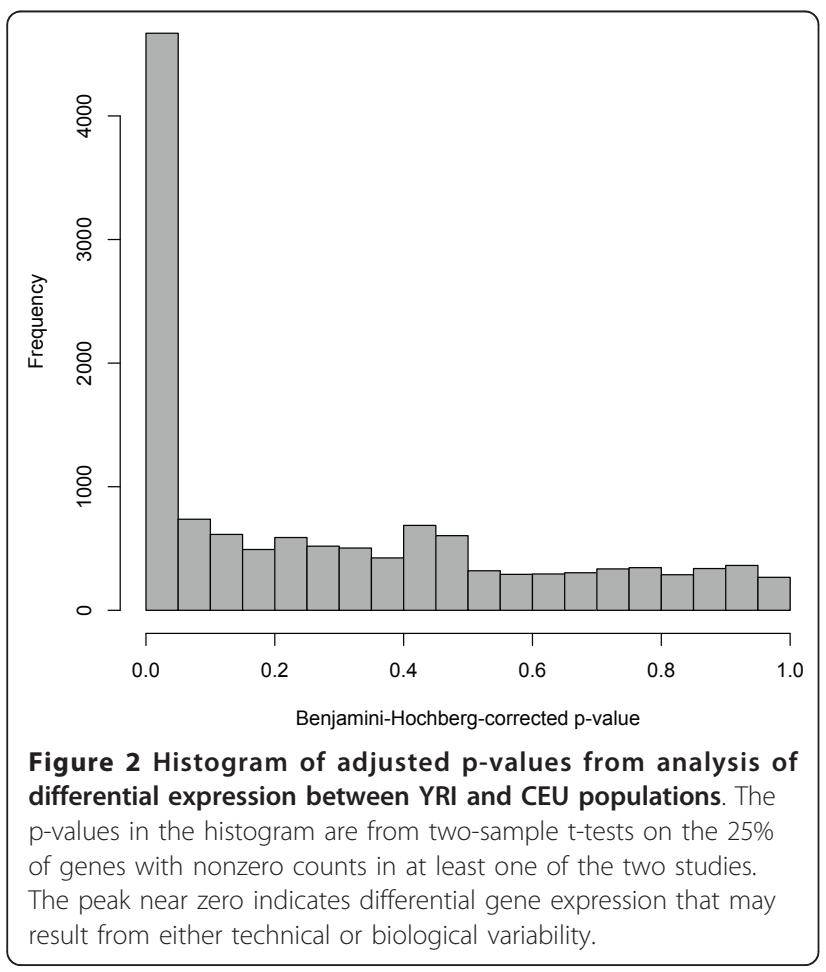

application may be to attempt to replicate findings from other studies (e.g., percentage of differentially expressed genes). Additionally, all commands and manifest files used in preprocessing are available on the website, so users can create their own count tables should they desire alternative parameterizations: e.g., alternative alignment parameters can be passed to Bowtie, the truncation length can be changed, or the pool-tech-reps option can be removed.

\section{Conclusions}

ReCount addresses two key issues for statistical researchers interested in RNA sequencing: (1) small sample sizes in many available studies and (2) computational difficulties in developing analysis-ready RNAsequencing data. By providing Myrna manifest files and $\mathrm{R}$ scripts that reproduce the count tables in ReCount, our database also allows for flexible exploration of a large number of organized RNA-sequencing datasets. We anticipate that ReCount will be useful to both the statistical and bioinformatics community as a resource for readily analyzable RNA-sequencing data.

\section{Availability and Requirements}

ReCount is publicly accessible at http://bowtie-bio.sf.net/ recount.

\section{Acknowledgements and Funding}

The authors would like to acknowledge discussions with Kasper Hansen and Rafael Irizarry. Support for this research was provided by an Amazon Web Services in Education Research Grant and a Bloomberg JHSPH Faculty Innovation Award to JL. BL was supported by NIH Grants P41-HG004059 and R01-HG005220. AF was supported by NIH Grant T32GM074906.

Authors' contributions

$J \mathrm{~L}$ and $\mathrm{BL}$ conceived the study. AF and BL contributed new methods and built the database. AF, JL and BL wrote the paper. All authors read and approved the final manuscript.

Competing interests

The authors declare that they have no competing interests.

Received: 18 August 2011 Accepted: 16 November 2011 Published: 16 November 2011

References

1. Wang Z, Gerstein M, Snyder M: RNA-Seq: a revolutionary tool for transcriptomics. Nat Rev Genet 2009, 10:57-63.

2. Stein LD: The case for cloud computing in genome informatics. Genome Biol 2010, 11:207.

3. Hansen KD, Wu Z, Irizarry RA, Leek JT: Sequencing technology does not eliminate biological variability. Nat Biotechnol 2011, 29(7):572-573.

4. Auer PL, Doerge RW: Statistical design and analysis of RNA sequencing data. Genetics 2010, 185:405-416.

5. Barrett T, Troup DB, Wilhite SE, Ledoux P, Evangelista C, Kim IF, Tomashevsky M, Marshall KA, Phillippy KH, Sherman PM, Muertter RN, Holko M, Ayanbule O, Yefanov A, Sobolera A: NCBI GEO: archive for functional genomics data sets - 10 years on. Nucleic Acids Res 2011, 39(suppl 1):D1005-D1010.

6. Gentleman RC, Carey VJ, Bates DM, Bolstad B, Dettling M, Dudoit S, Ellis B, Gautier L, Ge Y, Gentry J, Hornik K, Hothorn T, Huber W, lacus S, Irizarry R, 
Leisch F, Li C, Maechler M, Rossini AJ, Sawitzki G, Smith C, Smyth G, Tierney L, Yang JY, Zhang J: Bioconductor: open software development for computational biology and bioinformatics. Genome Biol 2004, 5(10): R80.

7. Human BodyMap 2.0 data from Illumina. 2011 [http://www.ensembl.info/ blog/2011/05/24/human-bodymap-2-0-data-from-illumina/].

8. Cheung VG, Nayak RR, Wang IX, Elwyn S, Cousins SM, Morley M, Spielman RS: Polymorphic cis- and trans-regulation of human gene expression. PLOS Biol 2010, 8(9):e1000480.

9. Core $L J$, Waterfall JJ, Lis JT: Nascent RNA sequencing reveals widespread pausing and divergent initiation at human promoters. Science 2008, 322(5909):1845-1848.

10. Blekhman R, Marioni JC, Zumbo P, Gilad Y: Sex-specific and lineagespecific alternative splicing in primates. Genome Res 2010, 20(2):180-189.

11. Bullard JH, Purdom E, Hansen KD, Dudoit S: Evaluation of statistical methods for normalization and differential expression in mRNA-Seq experiments. BMC bioinformatics 2010, 11:94.

12. Montgomery SB, Sammeth M, Gutierrez-Arcelus M, Lach RP, Ingle C, Nisbett J, Guigo R, Dermitzakis ET: Transcriptome genetics using second generation sequencing in a Caucasian population. Nature 2010, 464(7289):773-777.

13. Pickrell JK, Marioni JC, Pai AA, Degner JF, Engelhardt BE, Nkadori E, Veyrieras JB, Stephens M, Gilad Y, Pritchard JK: Understanding mechanisms underlying human gene expression variation with RNA sequencing. Nature 2010, 464(7289):768-772.

14. Sultan M, Schulz MH, Richard H, Magen A, Klingenhoff A, Scherf M, Seifert M, Borodina T, Soldatov A, Parkhomchuk D, Schmidt D, O'Keefe S, Haas S, Vingron M, Lehrach $H$, Yaspo ML: A global view of gene activity and alternative splicing by deep sequencing of the human transcriptome. Science 2008, 321(5891):956-960.

15. Wang ET, Sandberg R, Luo S, Khrebtukova I, Zhang L, Mayr C, Kingsmore SF, Schroth GP, Burge CB: Alternative isoform regulation in human tissue transcriptomes. Nature 2008, 456(7221):470-476.

16. Katz $Y$, Wang ET, Airoldi EM, Burge CB: Analysis and design of RNA sequencing experiments for identifying isoform regulation. Nat Methods 2010, 7(12):1009-1015.

17. Mortazavi A, Williams BA, McCue K, Schaeffer L, Wold B: Mapping and quantifying mammalian transcriptomes by RNA-Seq. Nat Methods 2008, 5(7):621-628.

18. Trapnell C, Williams BA, Pertea G, Mortazavi A, Kwan G, Van Baren MJ, Salzberg SL, Wold BJ, Pachter L: Transcript assembly and quantification by RNA-Seq reveals unannotated transcripts and isoform switching during cell differentiation. Nat Biotechnol 2010, 28(5):511-515.

19. Yang F, Babak T, Shendure J, Disteche CM: Global survey of escape from X inactivation by RNA-sequencing in mouse. Genome Res 2010, 20(5):614-622

20. Bottomly P, Walter NA, Hunter JE, Darakijian P, Kawane S, Buck KS, Searles RP, Mooney M, McWeeney SK, Hitzemann R: Evaluating gene expression in C57BL/6J and DBA/2J mouse striatum using RNA-Seq and microarrays. PLoS One 2011, 6(3):e17820.

21. Nagalakshmi U, Wang Z, Waern K, Shou C, Raha D, Gerstein M, Snyder M: The transcriptional landscape of the yeast genome defined by RNA sequencing. Science 2008, 320(5881):1344-1349.

22. Hammer $P$, Banck MS, Amberg R, Wang C, Petznick G, Luo S, Khrebtukova I, Schroth GP, Beyerlein P, Beutler AS: mRNA-seq with agnostic splice site discovery for nervous system transcriptomics tested in chronic pain. Genome Res 2010, 20(6):847-860.

23. Hillier LW, Reinke $V$, Green P, Hirst M, Marra MA, Waterston RH: Massively parallel sequencing of the polyadenylated transcriptome of $C$. elegans. Genome Res 2009, 19(4):657-666.

24. Graveley BR, Brooks AN, Carlson JW, Duff MO, Landolin JM, Yang L, Artieri CG, van Baren MJ, Boley N, Booth BW, Brown JB, Cherbas L, Davis CA, Dobin A, Li R, Lin W, Malone JH, Mattiuzzo NR, Miller D, Sturgill D, Tuch BB, Zaleski C, Zhang D, Blanchette, Dudoit S, Eads B, Green RE, Hammonds A, Jiang L, Kapranov P, Langton L, Perrimon N, Sandler JE, Wan KE, Willingham A, Zhang Y, Zou Y, Andrews J, Bickel PJ, Brenner SE, Brent MR, Cherbas P, Ginergas TR, Hoskins RA, Kaufman TC, Oliver B, Celniker SE: The developmental transcriptome of Drosophila melanogaster. Nature 2011, 471(7339):473-479.

25. Flicek P, Amode MR, Barrell D, Beal K, Brent S, Chen Y, Clapham P, Coates G, Fairley S, Fitzgerald S, Gordon L, Hendrix M, Hourlier T, Johnson N, Kahari A,
Keefe D, Keenan S, Kinsella R, Kokocinski F, Kulesha E, Larsson P, Longden I, McLaren W, Overduin B, Pritchard B, Riat HS, Rios D, Ritchie GR, Ruffier M, Schuster M, Sobral D, Spudich D, Tang YA, Trevanion S, Vandrovcova J, Vilella AJ, White S, Wilder SP, Zadissa A, Zamora J, Aken BL, Birney E, Cunningham F, Dunham I, Durbin R, Fernandez-Suarez XM, Herrero J, Hubbard TJ, Parker A, Proctor G, Vogel J, Searle SM: Ensembl 2011. Nucleic Acids Res 2011, 39(suppl 1):D800.

26. Langmead B, Hansen KD, Leek JT: Cloud-scale RNA-sequencing differential expression analysis with Myrna. Genome Biol 2010, 11(8):R83.

27. Leinonen $R$, Sugawara $H$, Shumway $M$ : The sequence read archive. Nucleic Acids Res 2011, 39(suppl 1):D19-D21.

28. Bolstad B, Irizarry R, Åstrand M, Speed T: A comparison of normalization methods for high density oligonucleotide array data based on variance and bias. Bioinformatics 2003, 19(2):185-193.

29. Benjamini $Y$, Hochberg $Y$ : Controlling the false discovery rate: a practical and powerful approach to multiple testing. Journal of the Royal Statistical Society. Series B (Methodological) 1995, 289-300.

30. Storey JD, Madeoy J, Strout JL, Wurfel M, Ronald J, Akey JM: Geneexpression variation within and among human populations. Am J Hum Genet 2007, 80(3):502-509.

31. Spielman RS, Bastone LA, Burdick JT, Morley M, Ewens WJ, Cheung VG: Common genetic variants account for differences in gene expression among ethnic groups. Nat Genet 2007, 39(2):226-231.

doi:10.1186/1471-2105-12-449

Cite this article as: Frazee et al:: ReCount: A multi-experiment resource of analysis-ready RNA-seq gene count datasets. BMC Bioinformatics 2011 $12: 449$

\section{Submit your next manuscript to BioMed Central and take full advantage of:}

- Convenient online submission

- Thorough peer review

- No space constraints or color figure charges

- Immediate publication on acceptance

- Inclusion in PubMed, CAS, Scopus and Google Scholar

- Research which is freely available for redistribution 